\title{
Development of Dual Beam High Speed Doppler OFDI
}

\author{
SunHee Kim*, TaeJin Park** and Wang-Yuhl $\mathrm{Oh}^{* *}{ }^{\dagger}$
}

\begin{abstract}
This paper describes development of a high speed Doppler OFDI system for non-invasive vascular imaging. Doppler OFDI (optical frequency domain imaging) is one of the phase-resolved second generation OCT (optical coherence tomography) techniques for high resolution imaging of moving elements in biological tissues. To achieve a phase-resolved imaging, two temporally separated measurements are required. In a conventional Doppler OCT, a pair of massively oversampled successive A-lines is used to minimize de-correlation noise at the expense of significant imaging speed reduction. To minimize a de-correlation noise between targeted two measurements without suffering from significant imaging speed reduction, several methods have been developed such as an optimized scanning pattern and polarization multiplexed dual beam scanning. This research represent novel imaging technique using frequency multiplexed dual beam illumination to measure exactly same position with aimed time interval. Developed system has been verified using a tissue phantom and mouse vessel imaging.
\end{abstract}

Keywords: Optical Coherence Tomography, Doppler OFDI, Noninvasive Imaging, Flow Speed Measurement

\section{Introduction}

The Optical coherence tomography (OCT) is non-invasive high speed $3 \mathrm{D}$ imaging using a broad band laser light source with the Michelson interferometer configuration. Optical frequency domain imaging (OFDI) is developed to overcome slow imaging speed of OCT, so called second generation OCT [1].

Among the OFDI system, the Doppler OFDI system has been used to detect moving elements using Doppler phenomena inside an imaging target such as vessel structure inside a tissue. For OFDI system, this Doppler shift appears as a phase shift value determined by moving velocity of particles and a time interval between two measurements [2-4]. Therefore, two measurements should be achieved at exactly same position with a temporal separation. This principle strictly limits performance of Doppler OCT as slight movement between two scan can increase phase noise significantly which disables

\section{Doppler shift detection [5-7].}

The Conventional Doppler OCT system used oversampling in $\mathrm{X}$ or $\mathrm{Y}$ scanning direction to minimize a transverse displacement of an imaging beam between two measurement points with a time interval which naturally reduces imaging speed.

Recently, several methods have been developed such as an optimized scanning pattern or polarization multiplexed dual beam system. The optimized scanning patterns such as a saw tooth scanning, segmented saw tooth scanning and step scanning are achieved by programmed scanning motor control [8,9]. This method could decrease de-correlation noise caused by un-wanted beam displacement significantly. However, mechanical limitation of the scanning mirror patterning could still limit imaging speed. The polarization multiplexed dual beam scanning method could increase the imaging speed up to general OCT system as two measurement points can be achieved with two beams without any

[Received: June 13, 2013, Revised: June 24, 2013, Accepted: June 25, 2013] *Korea Railroad Research Institute, \#176, Cheoldo bangmulgwan-ro, Uiwang, Gyeonggi-do, Korea **Department of Mechanical Engineering, Korea Advance Institute of Science and Technology, Daejeon 305-701, Korea †Corresponding Author: woh1@kaist.ac.kr

(c) 2013, Korean Society for Nondestructive Testing 
imaging speed loss [10-12]. However, a birefringence difference between two scanning beams could induce the additional phase noise which decreases a phase sensitivity of the system.

Therefore, this paper represents the novel dual beam scanning method to minimize the de-correlation noise while maintaining a high imaging speed and multi-functionality of the OFDI system using a frequency multiplexed dual beam illumination [13].

\section{Method}

\subsection{Principle of OFDI Imaging System}

Fig. 1 represents a configuration of the developed high speed Doppler OFDI system. A short cavity length wavelength-swept laser (WSL) with a tunable Fabry-Perot filter (Lambdaquest X1310) and delay lines for copy and paste technique provides $120 \mathrm{kHz}$ tuning speed over wavelength range of $140 \mathrm{~nm}$ centered at $1280 \mathrm{~nm}$. Polarization modulator is attached at laser output to develop multi-function OFDI system with an additional polarization sensitive imaging. WSL was coupled into a fiber-based
Michelson interferometer splitting into a sample arm and reference arm after splitting into fiber Bragg grating (FBG) by a $90 / 10$ fiber coupler to generate a trigger signal. A fiber pig tailed frequency shifter of $21.25 \mathrm{MHz}$ (Brimrose) is inserted to remove depth degeneracy.

A Fabry-Perot filter for wavelength-swept laser is synchronized with data-acquisition(DAQ) board using a master clock to tminimize the starting wavelength noise of the each A-line. Along with the Fabry-Perot driver and DAQ, two frequency shifter drivers are synchronized using interlocked $340 \mathrm{MHz}$ and $10 \mathrm{MHz}$ reference signals from frequency generator (Nova Source Co.) This configuration could minimize a depth dependent noise and depth independent noise of the phase difference between two temporally separated A-lines caused by the starting wavelength delay and frequency shifter clock delay for each A-line [4]. The X-axis galvanometer and $\mathrm{Y}$-axis motorized translation stage is used for a 3D imaging as the wavelength swept source could achieve a Z-axis imaging without depth scanning. The specific imaging optics configuration of the dual beam system is further described.

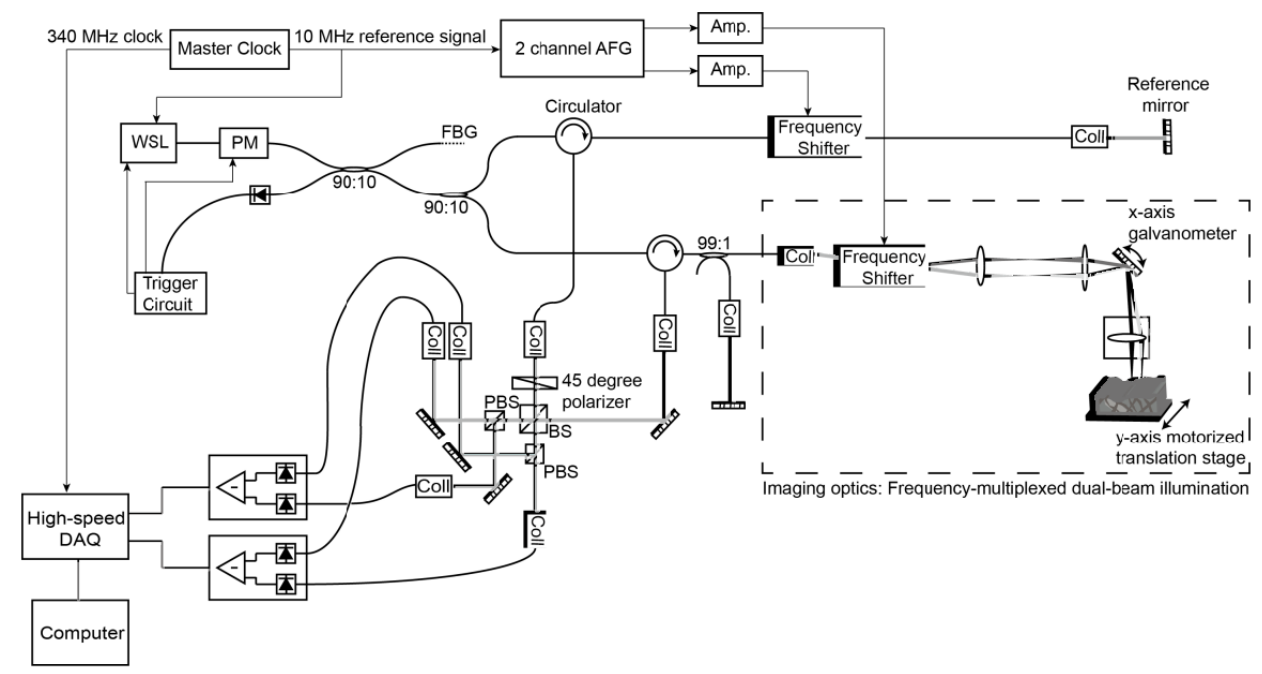

Fig. 1 Schematic diagram of the high-speed Doppler OFDI system. WSL: wavelength swept laser, PM: polarization modulator, Col: optical collimator, FS: frequency shifter, BS: beam splitter, PBS: polarizing beam splitter. FBG: fiber Bragg grating, DAQ: data acquisition unit 


\subsection{Dual Beam Doppler OFDI System}

Frequency-multiplexed dual beam illumination is achieved with an acousto-optic frequency shifter in the sample arm illustrated in Fig. 1 which angularly separates the zero-order (frequency un-shifted) and the first-order (frequency shifted) lights. A galvanometer mirror scanner with a rotation axis perpendicular to the plane of an illumination beams provides the beam scanning in the way that one of the angularly separated beams follows exactly the same path of the other. By carefully adjusting the scanning speed of the galvanometer, we can have two angularly separated beams illuminate the same location on the sample with a time interval corresponding to exact multiple of the A-line period, effectively removing phase de-correlation without slowing down the imaging speed. Since each beam is encoded with a distinct frequency shift, the two temporally separated beams are easily de-multiplexed in data processing.

Imaging optics part of the Fig. 1 shows specific diagram of dual beam configuration. Up-shifted beam is design to follow non-shifted beam along the $\mathrm{X}$ axis galvano-scanning mirror with calculated displacement. Beam size and incident angle of collimated beam to frequency shifter is adjusted before align lens system to minimize bandwidth loss through frequency shifter and divide power into two beam with calculated ratio. Spatial displacement between two beams on sample can be adjusted by controlling position of the scope system (lens1 and lens2) and scanning part with pre-set angular displacement of theta from the frequency shifter. Therefore we get mixed signal of non-shifted beam with up-shifted beam illuminating on different position on same time. Frequency multiplexing was implemented to separate them and apply on different time set.

To achieve frequency multiplexed dual beam illumination, we used frequency shifter to separate two beams without any optical alternation. Using two frequency shifters on reference arm and sample arm, we could separate two beams in frequency domain which is depth information in OFDI system without depth degeneracy. With $340 \mathrm{MHz}$ DAQ and $120 \mathrm{kHz}$ swept source with $140 \mathrm{~nm}$ bandwidth, we divided 0 to $85 \mathrm{MHz}$ for beam 1 and 85 to $170 \mathrm{MHz}$ for beam 2 with $4 \mathrm{~mm}$ depth for each. Motorized Y-axis stage could scan whole leg of the mouse and the brain without additional positioning of the mouse or sample to maximize bench top high speed Doppler imaging system function.

To minimize scanning mirror related additional de-correlation noise, we have controlled beam size and beam position on the galvano scanning mirror [4]. A Beam size of the light was limited up to $1 \mathrm{~mm}$ on the scanning mirror with precise position control on a center. Also, two angularly separatedbeams are positioned on the exactly same spot with different angle on the scanning mirror to remove additional noise. As Doppler phase shift data is calculated from the difference between two scanning beams, beam deformation effect caused by the scanning mirror cannot effect on the data in condition of the precise alignment as we described before.

\subsection{System Verification}

To represent phase sensitivity of the system, homogeneous phantom is used to measure phase sensitivity related to the SNR. As we developed system with reduced signal noise caused by de-correlation, phase noise should be limited by signal intensity as function of the inverse proportion of radial root value of the SNR.

De-correlation noise is caused by spatial difference between two measurement points which have unrelated phase information regardless Doppler signal from moving particle. This de-correlation noise is directly related to 


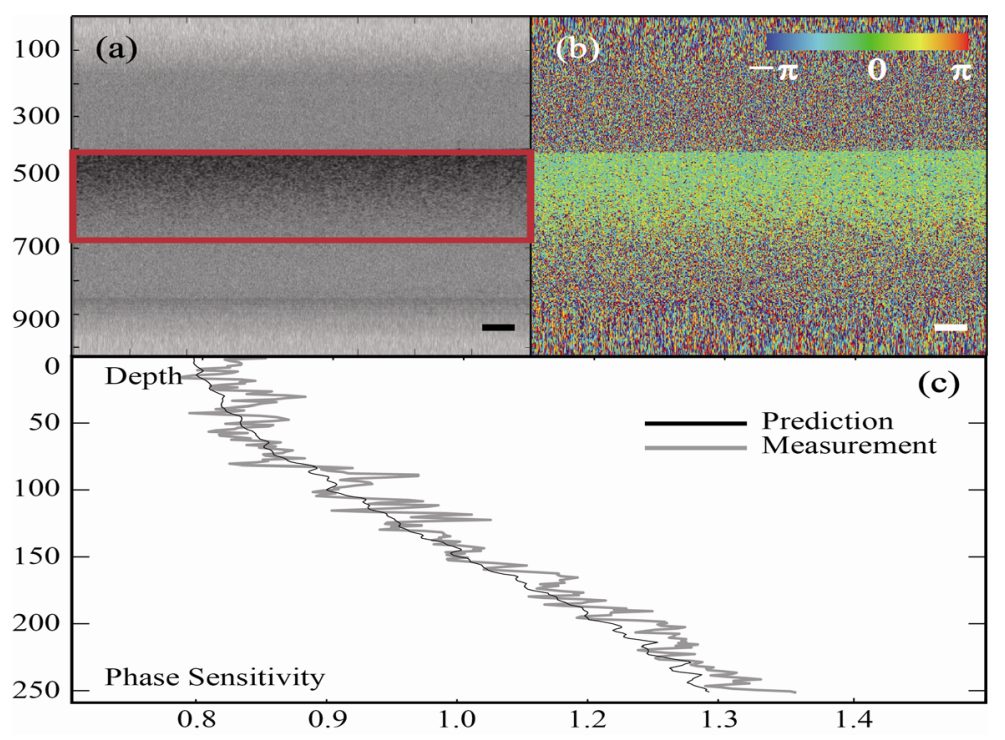

Fig. 2 (a) Intensity image of homogeneous phantom (b) phase difference image with two beams (c) phase noise graph according to the imaging depth in marked area with box. Black: SNR related phase noise standard deviation along the 800 A-lines with $20 \%$ de-correlation noise. Gray: Actual phase noise following theoretical prediction. (a), (b) shows $4 \mathrm{~mm}$ (depth) $\times 7 \mathrm{~mm}$ (transverse) image

the sensitivity of the phase difference measurement to calculate Doppler shift.

Phase noise calibration has been done before we measure phase noise relation with SNR to remove frequency shifter related depth independent noise and bulk motion noise by histogram based calibration method. Timing jitter noise between DAQ and WSL of each A-line is minimized by system synchronization as we described before.

To adjust histogram based calibration function applying on our system, intensity threshold filter and standard deviation of phase difference filter long the A-line have been applied. As you can see in Fig. 2, SNR decreases according to the depth and phase noise is following SNR decrease along the theoretical prediction based on the SNR limitation with portion of the de-correlation noise. This result could represent that measurement and calibration process could successfully minimize noise source caused by bulk motion and frequency shifter noise. However, $20 \%$ of de-correlation noise is still remained caused by alignment limitation and frequency shifter induced beam difference.

To maximize advantage of the high speed Doppler OFDI system, we have developed real time Doppler imaging platform to distinguish blood flow and flow rate. Signal from polarization diversity balanced detector is received by PX14400 digitizer and applied suggested histogram based calibration in Z800 workstation for processing. Real time display frame rate is 5 fps. Display processing is separated from detection and saving process to prevent data loss. Selected frame from dataset is processed and displayed.

To display high speed Doppler imaging functionality of the system, we have performed the $3 \mathrm{D}$ imaging of the vascular structure of the living mouse in-vivo sample with fabricated flow phantom.

Fig. $3(a, b)$ shows Doppler OFDI images of a flow phantom and a mouse vessel structure from different view point. Time interval between the two illumination beams is set to $0.26 \mathrm{msec}$ (32 A-line periods). High-speed vasculature 


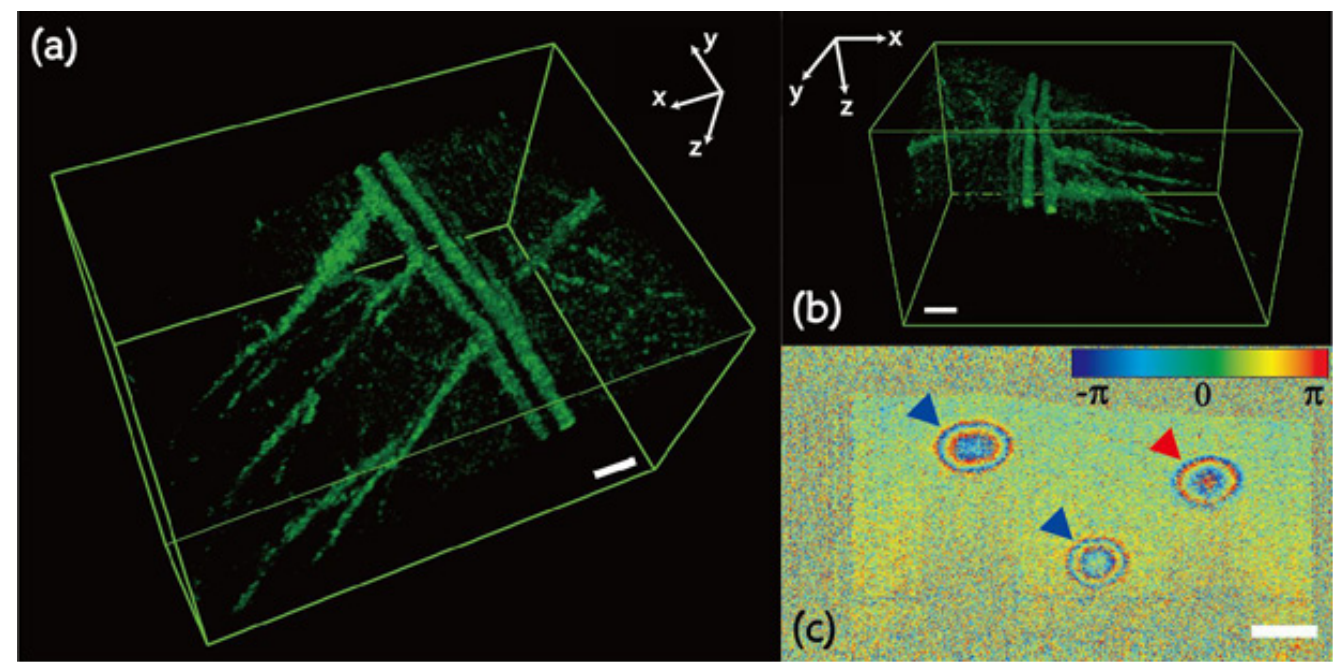

Fig. 3 3D micro-vasculature image. (a,b) mouse thigh vasculature $7 \mathrm{~mm} \times 3.7 \mathrm{~mm} \times 4 \mathrm{~mm}$ imaged in 4.41 seconds. (c) flow phantom image with 3 different rates of upward flow (blue arrow - left and center) and downward flow (red arrow - right). Scale bar: $500 \mu \mathrm{m}$

images of a mouse thigh in-vivo were obtained at 117.4 fps (1024 A-lines per frame) over $7 \mathrm{~mm} \times 4 \mathrm{~mm}$ (transverse $\times$ axial). Total imaging range was measured within 5 seconds with Doppler and intensity information. Fig. 3(c) shows laminar flow profile along the tube cross section. The measured flow speeds and the flow rates match well with the calculated values from the setting flow rate by the syringe pump which can demonstrate that Doppler imaging with developed system can display accurate flow velocity value with proper calibration as we described before.

This outstanding imaging speed can be compared with conventional OFDI imaging without Doppler information as conventional Doppler OCT cannot achieve phase difference calculation at this speed.

\section{Conclusions}

We demonstrate high-speed Doppler OFDI using frequency multiplexed dual-beam illumination. This novel scheme provides high-speed and large area Doppler OFDI imaging without suffering from phase de-correlation or slowing down imaging speed. To remove additional de-correlation noise, micro path length controller for each beam path should be further developed. We showed in-vivo blood flow image in mouse leg exhibiting femoral vascular network which was not visible in structural image with conventional intensity OFDI in same imaging speed. This shows developed device could be useful tool to image moving particle inside the targeted material such as living tissue for clinical use or other materials for industrial use.

\section{Acknowledgment}

This work supported by a NRF grant 2012-0005633 and High Risk High Return Project of KAIST.

\section{References}

[1] S. Makita, Y. Hong, M. Yamanari, T. Yatagai and Y. Yasuno, "Optical coherence angiography," Optics Express, Vol. 14, No. 17, pp. 7821-7840 (2006)

[2] Z. Chen, T. E. Milner, D. Dave and J. Stuart Nelson, "Optical Doppler tomo- 
graphic imaging of fluid flow velocity in highly scattering media," Optics Letters, Vol. 22, No. 1, pp. 64-66 (1997)

[3] Y. Zhao, Z. Chen, C. Saxer, S. Xiang, J. F. de Boer and J. S. Nelson, "Phaseresolved optical coherence tomography and optical Doppler tomography for imaging blood flow in human skin with fast scanning speed and high velocity sensitivity," Optics Letters, Vol. 25, Issue 2, pp. 114-116 (2000)

[4] B. Vakoc, S. Yun, J. de Boer, G. Tearney and B. Bouma, "Phase-resolved optical frequency domain imaging," Optics Express, Vol. 13, No. 14, pp. 5483-5493 (2005)

[5] B. J. Vakoc, G. J. Tearney and B. E. Bouma, "Statistical properties of phasedecorrelation in phase-resolved Doppler optical coherence tomography," IEEE Transactions on Medical Imaging, Vol. 28, No. 6, pp. 814-821 (2009)

[6] R. A. Leitgeb, L. Schmetterer, C. K. Hitzenberger, A. F. Fercher, F. Berisha, M. Wojtkowski and T. Bajraszewski, "Real-time measurement of in vitro flow by Fourier-domain color Doppler optical coherence tomography," Optics Letters, Vol. 29, Issue 2, pp. 171-173 (2004)

[7] S. H. Yun, G. Tearney, J. de Boer and B. Bouma, "Motion artifacts in optical coherence tomography with frequency-domain ranging," Optics Express, Vol. 12, Issue 13, pp. 2977-2998 (2004)

[8] B. J. Vakoc, R. M. Lanning, J. A. Tyrrell, T. P. Padera, L, A. Bartlett, T. Stylianopoulos, L. L. Munn, G. J. Tearney, D. Fukumura, R. K. Jain and B. E. Bouma, "Three-dimensional microscopy of the tumor microenvironment in vivo using optical frequency domain imaging," Nature Medicine, Vol. 15, No. 10, pp. 1219-1223 (2009)

[9] I. Grulkowski, I. Gorczynska, M. Szkulmowski, D. Szlag, A. Szkulmowska, R. A. Leitgeb, A. Kowalczyk and M. Wojtkowski, "Scanning protocols dedicated to smart velocity ranging in spectral OCT," Optics Express, Vol. 17, Issue 26, pp. 23736-23754 (2009)

[10] S. Makita, F. Jaillon, M. Yamanari and Y. Yasuno, "Dual-beam-scan Doppler optical coherence angiography for birefringenceartifact-free vasculature imaging," Optics Express, Vol. 20, Issue 3, pp. 2681-2692 (2012)

[11] S. Makita, F. Jaillon, M. Yamanari, M. Miura and Y. Yasuno, "Comprehensive in vivo micro-vascular imaging of the human eye by dual-beam-scan Doppler optical coherence angiography," Optics Express, Vol. 19, Issue 2, pp. 1271-1283 (2011)

[12] S. Zotter, M. Pircher, T. Torzicky, M. Bonesi, E. Götzinger, R. A. Leitgeb and C. K. Hitzenberger, "Visualization of microvasculature by dual-beam phaseresolved Doppler optical coherence tomography," Optics Express, Vol. 19, Issue 2, pp. 1217-1227 (2011)

[13] W. Y. Oh, S. H. Yun, B. J. Vakoc, M. Shishkov, A. E. Desjardins, B. H. Park, J. F. de Boer, G. J. Tearney and B. E. Bouma, "High-speed polarization sensitive optical frequency domain imaging with frequency multiplexing," Optics Express, Vol. 16, No. 2, pp. 1096-1103 (2008) 